DOI 10.3233/FI-2010-212

IOS Press

\title{
Special Issue on Intelligent Data Analysis in Granular Computing
}

\author{
Preface
}

Granular Computing $(\mathrm{GrC})$ is a general computation theory for effectively using granules such as classes, clusters, subsets, groups, intervals and rules to build an efficient computational model for complex applications with huge amounts of data, information and knowledge. Though the label is relatively recent, the basic notion and principle of granular computing have appeared in many related fields, such as information hiding in programming, granularity in artificial intelligence, divide-and-conquer in theoretical computer science, interval computing, cluster analysis, fuzzy and rough set theories, neutrosophic computing, quotient space theory, belief functions, machine learning, databases, and many others.

This special issue focuses on both research and application aspects about granular computing, especially on intelligent data analysis. There are totally eight papers included, each of which has received outstanding recommendations from the reviewers and has been revised by authors for this publication. Their contents are briefly introduced below.

The first paper presents a study of three genetic association rules extraction methods to show their effectiveness for mining quantitative association rules. The second paper proposes a scalable 2-leveled approximated hyper-image-based clustering framework for mining local-correlated clusters from high dimensional datasets. Then a mobile network monitoring system based on spatio-temporal data mining has been designed in the third paper. The fourth paper states a cost-effective methodology for the prediction of demographic website visitor profiles that can be used for on-line advertising targeting purposes. The fifth paper aims to investigate the possibility of detecting outliers in correlated subspaces, with a novel approach proposed. Besides, the sixth paper investigates the application of evolution strategies to Nadaraya-Watson based kernel regression. This seventh paper proposes an application of data mining to medical risk management in clinical environments. The last paper proposes a model for multi-layered or chained situations and applies it to determining communication errors occurring in medical accidents and incidents. We are sure that you will find it a valuable resource in the development of new advanced intelligent systems. 
We are grateful to all the authors for their contributions and the referees for their vision and efforts. We would also like to express our thanks to Professor Andrzej Skowron, the editor-in-chief of the journal, for his great support to realize the special issue.

\section{The Guest Editors}

Shusaku Tsumoto - Shimane University, Japan

Tzung-Pei Hong - National University of Kaohsiung, Taiwan 